\title{
A novel wet-lab teaching model for trabeculectomy surgery
}

\author{
A. M. Porteous ${ }^{1} \cdot$ F. Ahmed ${ }^{1}$
}

Received: 15 February 2018 / Accepted: 28 February 2018 / Published online: 26 April 2018

(c) The Royal College of Ophthalmologists 2018

\section{Introduction}

Despite the recent advances in minimally invasive glaucoma surgery trabeculectomy remains the gold standard surgical technique for glaucoma that is refractory to medical therapy. Following the refinement of the surgical technique good trabeculectomy outcomes with low rates of surgical complications can now be achieved, but intensive proactive postoperative care is often required [1].

Mastering these new techniques requires time and practice, but the number of trabeculectomy operations being performed in the UK is reducing [2], so access to surgical training for trainees is becoming more challenging. Using simulation and wet-lab models to enhance learning is therefore becoming increasingly important. Such models are commonplace in cataract surgery [3] but are limited for glaucoma surgery. Those models previously described include a formalin-prepared pig eye model [4]; however, this requires the sourcing of materials that can potentially be difficult to access and expensive. We therefore propose a new novel wet-lab model that is inexpensive and easily accessible using a green apple, cling film and disposable instruments.

\section{Methods}

The equipment along with the steps required are detailed in Fig. 1.

The firm consistency of the skin and cortex of a green apple lends itself to the creation of the scleral flap as the tissue does not easily disintegrate, and when the flap is opened the thickness and uniformity of the flap can be assessed. As the apple surface is curved like

F. Ahmed

faisal.ahmed5@nhs.net

1 Western Eye Hospital, 153-173 Marylebone Road, NW1 5QH London, UK an eye the trainee can practice tilting the crescent blade to ensure the curvature is followed when extending sideways (Fig. 2).

\section{Discussion}

The benefits of this model are as follows: it is cost-effective and safe and can be used in both a clinical and non-clinical environment with or without the use of an operating microscope. It is not meant to be an accurate recreation of a human eye and there are models available that are designed to recreate this. The aim of this model is to provide a cheap and easily repeatable platform that any trainee can set-up and use in any situation that can recreate the key steps involved in the surgery.

This model not only allows glaucoma trainees to practice the steps of a trabeculectomy but can also be used as an aid to practice scleral suturing in cases of traumatic scleral or corneal perforations and closing of sclerostomies, in addition to practicing conjunctival suturing and closure with the use of the cling-film. Formative assessments could also potentially be undertaken on suture technique.

Model eyes and simulators are invaluable surgical tools for trainees and senior surgeons learning new techniques. We feel our apple model is a cost-effective tool for surgeons to practice their scleral and conjunctival incisions and suturing. A key aspect of learning a new surgical technique is repeated deliberate practice [5], therefore having a model that is easy to set-up and practice techniques repeatedly leads to an improvement in muscle memory and could potentially shorten the learning curve and importantly maximise the learning potential when starting to perform trabeculectomy surgery on patients. 
Fig. 1 Equipment and steps required

Fig. 2 a Prepared apple model detailing limbal marks and cling-film, $\mathbf{b}$ creation of the scleral flap using the crescent blade, $\mathbf{c}$ placement of releasable suture, $\mathbf{d}$ placement of conjunctival suture

\begin{tabular}{|l|}
\multicolumn{2}{|c|}{ Equipment } \\
- Disposable crescent blade \\
- Disposable $15 \quad$ degree \\
blade \\
- Disposable spring scissors \\
- Disposable needle holder \\
- Disposable tying forceps \\
- forceps \\
- Green apple \\
- Cling film \\
- Suture material (10.0 nylon \\
if using microscope, 8.0 \\
nylon if not) \\
\hline
\end{tabular}

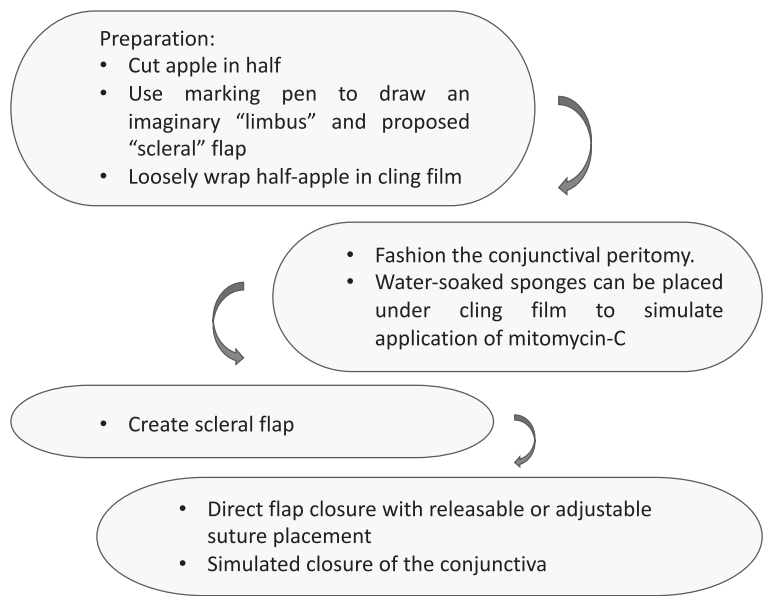


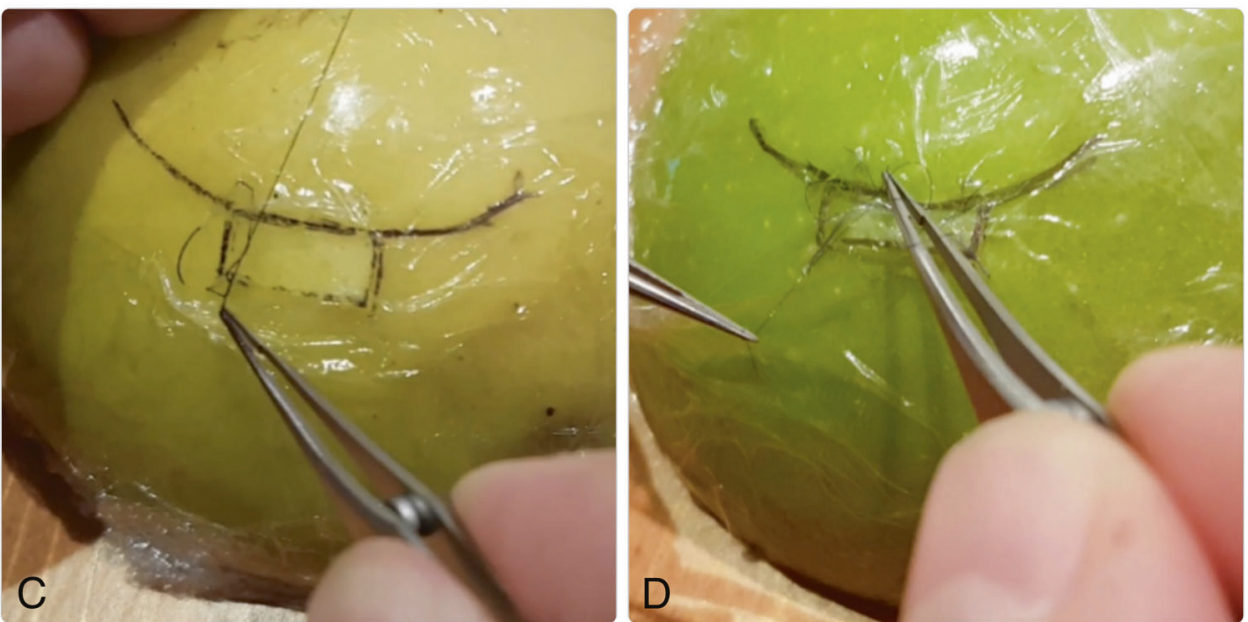

\section{Compliance with ethical standards}

Conflict of interest The authors declare that they have no conflict of interest.

\section{References}

1. Kirwan JF, Lockwood AJ, et al. Trabeculectomy in the $21^{\text {st }}$ century: a multicenter analysis. Ophthalmology. 2013;120:2532-9.

2. Murphy C, Ogston S, Cobb C, MacEwan C. Recent trends in glaucoma surgery in Scotland, England and Wales. Br J Ophthalmol. 2015;99:308-12. 
3. Thomsen AS, Kilgaard JF, Kjaerbo H, La Cour M, Konge L. Simulation-based certification for cataract surgery. Acta Ophthalmol. 2015;93:416-21.
4. Lee GA, Chiang MY-M, Shah P. Pig eye trabeculectomy - a wetlab teaching model. Eye. 2006;20:32-37.

5. Kavic MS. Teaching and learning of surgery. J Soc Laparoendosc Surg. 2012;16:341-4.

\title{
Are patients with poorer vision more polite? A study examining door closing tendencies in patients with poor vision
}

\author{
Sidath Wijetilleka ${ }^{1} \cdot$ Gwyn Williams $^{1}$ \\ Received: 13 December 2017 / Accepted: 14 March 2018 / Published online: 1 May 2018 \\ (c) The Royal College of Ophthalmologists 2018
}

With many changes to patient flow and the minimisation of unnecessary movement in patient treatment pathways becoming more important in planning new ophthalmic service delivery, it is easy to forget that there are individual variations between patient groups that are often overlooked. It was noticed that patients attending Singleton Hospital with worse vision tended to make a point of closing the clinic room door more consistently at the end of the consultation than those with better vision. If this relationship were found to be significant then tailored care could then be provided based on predicted behaviour. Patients who close the door themselves, which we will call 'polite' patients for the purpose of this study, may benefit from having their door closing tendencies taken into consideration in optimising service delivery and patient flow. It would also be interesting to know if patients with poorer vision are in fact 'politer' than their better seeing counterparts. There is no previously published study that has looked at this phenomenon before.

The clinic rooms at Singleton are designed with the patient closest to the door so the natural tendency is for the patient, upon completing the consultation, to reach the door first. A patient was deemed 'polite' if they closed the door behind them, or at least attempted to, while those needing assistance or for whatever reason were escorted to the door by the clinician were excluded from the study. All patients were seen in a number of medical retina clinics by one of two ophthalmologists. No invitation to close the door was

Sidath Wijetilleka

sidwijetilleka@doctors.net.uk given. A better seeing vs poor seeing patient was defined as vision worse than or better than 0.3 LogMAR (6/12 Snellen).

Over the course of the study 300 consultations were eligible for inclusion, of which 138 people had vision better than 0.3 LohMAR in their better eye; out of these only 40 closed the door behind them (29\%). There were 162 patients with vision worse than $0.3 \operatorname{LogMAR}$ in their better eye and out of these 138 closed the door behind them $(85 \%)$. This result was statistically significant with $p<0.05$ with statistical analysis via $\chi^{2}$ test.

It is interesting to hypothesise as to why patients with poorer vision are 'politer', according to our study. Perhaps it is a case of patients with better vision not being so attuned to how their actions affect the people or the environment around them. Perhaps patients with worse vision are accustomed to adapting their home circumstances such that unexpected variations may lead to trips or falls. Whatever the reason this is the first study that has demonstrated this outcome and this knowledge of expected patient behaviour may be important in helping us plan eye services. For example patients attending a low vision clinic may benefit from having the door propped open to avoid potential struggles with door closing upon leaving. It is also an important anthropological finding that independent to this is worthy of further study.

\section{Compliance with ethical standards}

Conflict of interest The authors declare that they have no conflict of interest.

$1 \quad$ Singleton Hospital, Swansea, UK 


\title{
HLA alleles in British Caucasians with mucous membrane pemphigoid
}

\author{
F. Hübner ${ }^{1} \cdot$ J. Setterfield ${ }^{2} \cdot$ A. Recke ${ }^{1} \cdot$ D. Zillikens ${ }^{1} \cdot$ E. Schmidt ${ }^{1,3} \cdot$ J. Dart $^{4}$ • \\ S. Ibrahim ${ }^{3}$ - the MMP Study Group 2009
}

Received: 12 January 2018 / Accepted: 17 January 2018 / Published online: 10 May 2018

(c) The Royal College of Ophthalmologists 2018

Mucous membrane pemphigoid (MMP) is an autoimmune mucosal scarring disease having severe ocular morbidity [1]. Disease susceptibility is associated with increased frequencies of human leukocyte antigen (HLA)-DQB1*03:01, HLA-DRB $1 * 11$, and HLA-DRB $1 * 04$ and decreased frequencies of DQB1*02 [2]. To explore correlations between clinical involvement and HLA-class-II alleles, we prospectively phenotyped a cohort of 55 British MMP patients, and $41 \mathrm{age/sex}$-matched controls (ethics approval reference 09/H0721/54).

Inclusion criteria have been described [3] and were preagreed clinical criteria with or without a positive direct immunofluorescence (DIF) study. HLA-typing used allelespecific sequencing protocols (Protrans S3 HLA-DRB1* and HLA-DQB1* Cyclerstrips, Protrans, Hockenheim, Germany). Statistical analysis used Fisher's exact test $(p)$, with Benjamini-Hochberg correction $\left(p_{\mathrm{c}}\right)$ defined as significant when $p_{\mathrm{c}}<0.05$.

The submission complies with the tenets of the Declaration of Helsinki and patients have provided appropriate ethical approval for publication.

Electronic supplementary material The online version of this article (https://doi.org/10.1038/s41433-018-0092-5) contains supplementary material, which is available to authorized users.

\section{S. Ibrahim}

saleh.ibrahim@uksh.de

1 Department of Dermatology, University of Lübeck, Lübeck, Germany

2 Guy's and St Thomas's NHS Foundation Trust and King's College London, London, UK

3 The Lübeck Institut of Experimental Dermatology (LIED), University of Lübeck, Lübeck, Germany

4 National Institute of Health Research (NIHR) Biomedical Research Centre at Moorfields Eye Hospital NHS Foundation Trust and UCL Institute of Ophthalmology, London, UK
The study dataset, with clinical and laboratory results, is provided in Table and Legend S1. Fifteen patients, 14 of them with ocular involvement, were DIF-negative of whom seven had antibodies to basement membrane zone epitopes. MMP-affected sites varied: eight were ocular only, ten oral only, 15 oral and ocular only and 22 multisite. Exons 2 and 3 in HLA-DQB1 were fully analysed in 54/55 patients and $39 / 41$ controls (Table 1a). The frequency of HLADQB1*03:01 was increased $\left(p_{\mathrm{c}}=<0.01\right)$ in 36/54 (67\%) of MMP patients (13 homozygous and 23 heterozygous) compared to 13/39 (33\%) controls (two homozygous and 11 heterozygous, Table 1a). Exon 2 of HLA-DRB1 was also fully analysed in 54/55 patients and 40/41 controls; HLADRB1*03:01 was decreased $\left(p=<0.01, \quad p_{\mathrm{c}}=0.045\right.$, Table 1b). Additionally, we compared the frequency of HLA-DQB $1 * 0301$ with controls for different sites of

Table 1a Distribution of HLA-DQB1 among patients and controls

$\frac{\text { Patients }}{(n=54) \text { in } \%} \frac{\text { Controls }}{(\mathrm{n}=39) \text { in } \%} p$-value $p_{\mathrm{c}}$

\begin{tabular}{|c|c|c|c|c|c|c|}
\hline \multicolumn{7}{|l|}{$H L A-D Q B 1$} \\
\hline HLA-DQB $1 * 02$ & 9 & 16.6 & 18 & 46.2 & $<0.01$ & $<0.01$ \\
\hline HLA-DQB1*03 & 48 & 88.9 & 20 & 51.3 & $<0.01$ & $<0.01$ \\
\hline HLA-DQB1*04 & 4 & 7.4 & 6 & 15.4 & 0.31 & 0.39 \\
\hline HLA-DQB1*05 & 13 & 24 & 11 & 28.2 & 0.81 & 0.81 \\
\hline HLA-DQB1*06 & 13 & 24 & 18 & 46.2 & 0.04 & 0.07 \\
\hline \multicolumn{7}{|l|}{$H L A-D Q B 1 * 02$} \\
\hline HLA-DQB $1 * 02: 01$ & 6 & 11.1 & 9 & 23.1 & 0.15 & ND \\
\hline HLA-DQB $1 * 02: 02$ & 3 & 5.5 & 7 & 17.9 & 0.09 & ND \\
\hline \multicolumn{7}{|l|}{$H L A-D Q B 1 * 03$} \\
\hline HLA-DQB $1 * 03: 01$ & 36 & 66.6 & 13 & 32.1 & $<0.01$ & 0.01 \\
\hline HLA-DQB $1 * 03: 02$ & 7 & 13 & 2 & 5.1 & 0.29 & 0.63 \\
\hline HLA-DQB $1 * 03: 03$ & 4 & 7.4 & 2 & 5.1 & 1 & 1 \\
\hline HLA-DQB1*03:05 & 0 & 0 & 1 & 2.6 & 0.42 & 0.63 \\
\hline HLA-DQB $1 * 03: 19$ & 1 & 1.8 & 0 & 0 & 1 & 1 \\
\hline HLA-DQB $1 * 03: 22$ & 0 & 0 & 1 & 1.3 & 0.42 & 0.63 \\
\hline
\end{tabular}


Table 1b Distribution of HLA-DRB1 among patients and controls

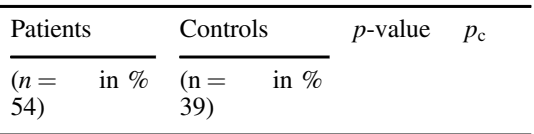

HLA-DRBI

\begin{tabular}{|c|c|c|c|c|c|c|}
\hline HLA-DRB $1 * 01$ & 10 & 18.5 & 12 & 30 & 0.23 & 0.48 \\
\hline HLA-DRB $1 * 0301^{\mathrm{a}}$ & 7 & 13 & 15 & 37.5 & $<0.01$ & 0.0 \\
\hline HLA-DRB1*04 & 20 & 37 & 7 & 17.5 & 0.04 & 0.15 \\
\hline HLA-DRB $1 * 05$ & 0 & 0 & 1 & 2.5 & 0.43 & 0.59 \\
\hline HLA-DRB1*07 & 5 & 9.3 & 10 & 25 & 0.05 & 0.14 \\
\hline HLA-DRB $1 * 08$ & 3 & 5.5 & 4 & 10 & 0.45 & 0.59 \\
\hline HLA-DRB $1 * 09$ & 3 & 5.5 & 0 & 0 & 0.27 & 0.48 \\
\hline HLA-DRB $1 * 11$ & 25 & 40.7 & 7 & 17.5 & $<0.01$ & 0.04 \\
\hline HLA-DRB $1 * 12$ & 3 & 5.5 & 1 & 2.5 & 0.63 & 0.75 \\
\hline HLA-DRB $1 * 13$ & 13 & 24 & 6 & 17.5 & 0.31 & 0.5 \\
\hline HLA-DRB1*14 & 1 & 1.8 & 0 & 0 & 1 & 1 \\
\hline HLA-DRB $1 * 15$ & 9 & 16.6 & 14 & 35 & 0.05 & 0.14 \\
\hline HLA-DRB1*16 & 1 & 1.8 & 0 & 0 & 1 & 1 \\
\hline \multicolumn{7}{|l|}{$H L A-D R B 1 * 11$} \\
\hline HLA- DRB1*11:01/11:97 & 12 & 22.2 & 5 & 12.5 & 0.29 & ND \\
\hline $\begin{array}{l}\text { HLA-DRB1* } \\
\text { 11:01/11:97 or } 11: 04\end{array}$ & 2 & 3.7 & 0 & 0 & 0.5 & ND \\
\hline $\begin{array}{l}\text { HLA-DRB } 1 * 11: 02 \text { or } \\
11: 36 \text { or } 11: 48\end{array}$ & 1 & 1.85 & 0 & 0 & 1 & ND \\
\hline HLA-DRB $1 * 11: 03$ & 2 & 3.7 & 0 & 0 & 0.5 & ND \\
\hline $\begin{array}{l}\text { HLA-DRB } 1 * 11: 03 \text { or } \\
11: 11\end{array}$ & 2 & 3.7 & 0 & 0 & 0.5 & ND \\
\hline HLA-DRB $1 * 11: 04$ & 6 & 11.1 & 2 & 5 & 0.46 & ND \\
\hline
\end{tabular}

In the first step of the analysis the gene frequencies for the allel groups of HLA-DQB1 (2-6) and HLA-DRB1 (1, 3-5, 7-9, and 11-16) were compared and in the second step, only significant alleles were further analysed regarding specific HLA-protein (e.g., HLA-DRB1*11) and compared. The $p$-value is given for comparisons of HLA gene frequencies, between cases and controls, using Fisher's exact test for each allele. The $p_{\mathrm{c}}$ value is the probability value after using the Benjamini-Hochberg correction for multiple testing.

$N D$ not done

a All patients with HLA-DRB $1 * 03$ expressed the specific protein HLA-DRB $1 * 0301$.

involvement showing that HLA-DQB $1 * 0301$ was increased in all subgroups except for ocular only MMP. Compared to controls, DIF-positive MMP had significantly increased HLA-DQB $1 * 0301(p=0.00009)$ but this difference was not significant for either DIF-negative MMP ( $p=0.113$ ) or for DIF-positive ocular only MMP.

Some studies have described a correlation of HLADQB1*03:01 with ocular and oral MMP or in ocular only MMP, whereas others have shown HLA-DQB 1*03:01 to be associated with multisite MMP [2]. In this study, the association of HLA-DQB $1 * 0301$ with MMP was lower than in the largest reported study [2] $\left(p_{\mathrm{c}}<0.00000028\right)$, possibly due to our inclusion of eight patients without detectable tissue-bound or serum antibodies, who were excluded from the latter study [2]. In ocular only MMP 50\% are DIF-negative but have a phenotype that both progresses and responds to therapy in the same way as DIF-positive cases [3, 4]. DIF-negative ocular MMP may result from inadequate test sensitivity, because of the small volumes of tissue involved, to dominance of an autoreactive T-cell mediated over a autoantibody-mediated disease [4] or because this is a different disease subset. We included our DIF-negative cases because to leave these out of this analysis disregards a group of cases which do not fit criteria for any other disease.

In our prospectively characterized cohort the association with HLA-DQB1*0301, HLA-DQB $1 * 02$, and HLADRB $1 * 11$ was corroborated, whereas HLA-DRB1*0301 was identified as a potentially protective allele, which requires confirmation in a larger study.

Funding From anonymous donors to John Dart's undesignated research funds, through a Grant from the Moorfields Eye Hospital Special Trustees MEH Special Trustees Reference ST $1111 \mathrm{~B}$ and from the NIHR Biomedical Research Centre for Ophthalmology, Major Award to support translational research initiatives (Ref.: BMRC 045). Part of J.D. salary is funded by the National Institute for Health Research (NIHR) Biomedical Research Centre at the Moorfields Eye Hospital NHS Foundation Trust and UCL Institute of Ophthalmology.

MMP Study Group 2009 Moorfields Eye Hospital, London: Debbie Booth, Elaina Reid, Nicole Carnt, Stefano Gugliemetti, Vijay Shanmuganathan, Martin Watson, Valerie Saw, Mark Wilkins, Vicky McCudden, Saj Ahmad,Catey Bunce. The UCL Institute of Ophthalmology, London: Virginia Calder, Waseem Naushin, Beverly Scott. Guys and Thomas's Hospital, London: Stephen Challacombe, Mike Gleeson, Sarah Ali, William Gage. Epivision (Ophthalmic Epidemiology Consultants), Penn, UK: Darwin Minassian. The Royal National ENT Hospital, London: Valerie Lund, Guri Sandhu.

\section{Compliance with ethical standards}

Conflict of interest The authors declare that they have no conflict of interest.

\section{References}

1. Dart J. Cicatricial pemphigoid and dry eye. Semin Ophthalmol. 2005;20:95-100.

2. Setterfield J, Theron J, Vaughan RW, et al. Mucous membrane pemphigoid: HLA-DQB1*0301 is associated with all clinical sites of involvement and may be linked to antibasement membrane $\mathrm{IgG}$ production. Br J Dermatol. 2001;145:406-14.

3. Ong HS, Setterfield JF, Minassian DC, Dart JK. The MMP Study Group 2009-14 Mucous membrane pemphigoid with ocular involvement: the clinical phenotype and its relationship to direct immunofluorescence findings. Ophthalmology. 2018;125:496-504.

4. Dart JKG. The 2016 Bowman Lecture. Conjunctival curses: scarring conjunctivitis 30 years on. Eye (Lond). 2017;31:301-32. https://doi.org/10.1038/eye.2016.284 


\title{
Retinal findings in a patient with mutations in $A B C C 6$ and $A B C A 4$
}

\author{
Omar A. Mahroo ${ }^{1,2,3} \cdot$ Kaoru Fujinami ${ }^{1,2,4} \cdot$ Anthony T. Moore ${ }^{1,2,5} \cdot$ Andrew R. Webster ${ }^{1,2}$
}

Received: 6 April 2018 / Accepted: 16 April 2018 / Published online: 16 May 2018

(c) The Royal College of Ophthalmologists 2018

In 2012, a case was reported in this journal, from our service [1], of a 24-year-old male of Somali ancestry (from consanguineous parents), referred initially due to reduced central vision. Fundus autofluorescence (FAF) showed angioid streaks but also a reduced signal in the central macula indicative of retinal pigment epithelium atrophy (Fig. 1). Severe macular dysfunction and generalised retinal involvement were shown on electrophysiological testing. Skin biopsy was consistent with a mild form of pseudoxanthoma elasticum (PXE) and a homozygous mutation in exon 7 of the ABCC6 gene (c.708_709dupCT, p. (Trp237fsX21)) was subsequently found. The severity, early onset and distribution of maculopathy were atypical for PXE and considered worthy of report. The purpose of the present correspondence is to provide an update to the case's interpretation.

Given the appearance of the maculopathy, the possibility of additional ABCA4-retinopathy was considered, particularly homozygosity for c.5882G $>$ A (p.G1961E) [2-5], a common allele in the Somali population [5]. Subsequently, PCR-amplification and Sanger sequencing of Exon 42 of $A B C A 4$ confirmed homozygosity for this allele. Later, DNA was tested for mutations in a number of genes implicated in macular dystrophies (Stargardt/Macular

These authors contributed equally: Omar A. Mahroo, Kaoru Fujinami.

Andrew R. Webster

Andrew.webster@ucl.ac.uk

1 UCL Institute of Ophthalmology, 11-43 Bath Street, London EC1V 9EL, UK

2 Moorfields Eye Hospital, 162 City Road, London EC1V 2PD, UK

3 Department of Ophthalmology, King's College London, St Thomas' Hospital Campus, Westminster Bridge Road, London SE1 7EH, UK

4 National Institute of Sensory Organs, Tokyo, Japan

5 Department of Ophthalmology, UCSF School of Medicine, San Francisco, CA 94143, USA dystrophy SmartPanel v5; Molecular Vision Laboratory, Hillsboro, Oregon), and the findings were confirmed, with no additional pathogenic mutations identified. Thus, this patient has bi-allelic variants in both $A B C C 6$ and $A B C A 4$, and the phenotype includes features of both, with the maculopathy more likely to be $A B C A 4$-related. Figure 1 depicts FAF imaging when the patient was 31 years old, and also ultra-widefield FAF imaging 9 years later. There has been mild enlargement of the areas of hypoautofluorescence (both in the central macula and the peripapillary angioid streaks); although, the peripheral retina appears unaffected.

Patients homozygous for this $A B C A 4$ mutation have been reported previously to have limited retinal disease with no peripheral involvement [2-4]. Our patient's ultra-widefield imaging appears to fit with this phenotype, although electrophysiological testing did show evidence of generalised retinal dysfunction as detailed in the first report [1], and the PXE might be contributory. Generalised retinal dysfunction in PXE has been previously reported [6].

It is tempting to speculate that the two distinct molecular pathologies might interact. The c.5882G>A (p.G1961E) $\mathrm{ABCA} 4$ allele is too prevalent in the general population (http://gnomad.broadinstitute.org/variant/1-94473807-C-T) to be a fully penetrant allele and so other modifying factors are likely to be acting. It is possible that many from Somalia with this $A B C A 4$ genotype remain normally sighted, but that, in this case, the additional compromise of RPE and/or photoreceptor function due to mineralisation of Bruch's from PXE, might contribute to early visual dysfunction.

The case is of particular interest: (i) it reminds clinicians of the possible co-occurrence of two unlinked recessive disorders in consanguineous families; (ii) it demonstrates that in some cases, a person's ethnic background can efficiently direct molecular testing (in this case a specific DNA base substitution in a single gene was suggested and confirmed using a single-amplimer PCR reaction in the laboratory); (iii) there remains no evidence that PXE can 

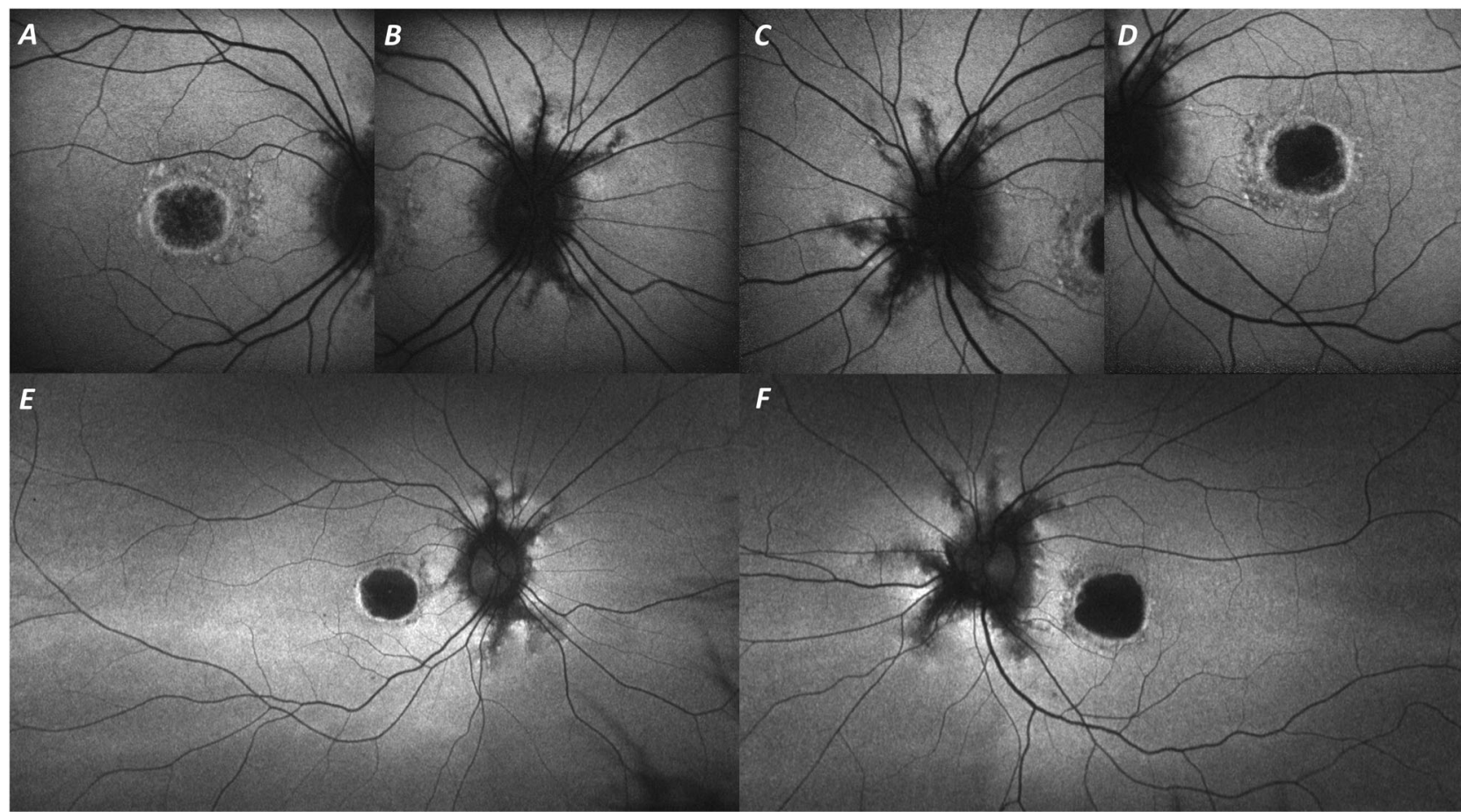

Fig. 1 Fundus autofluorescence findings 9 years apart. a-d Short wavelength $(488 \mathrm{~nm})$ autofluorescence imaging (Spectralis, Heidelberg, Germany) obtained age 31 . Angioid streaks are visible, as well as central macular hypo-autofluorescence (with a surrounding

produce a maculopathy that resembles that seen in Stargardt disease.

Funding NIHR Biomedical Research Centre at Moorfields Eye Hospital and the UCL Institute of Ophthalmology; Wellcome Trust (206619/Z/17/Z).

\section{Compliance with ethical standards}

Conflict of interest The authors declare that they have no conflict of interest.

\section{References}

1. Tan MH, Vanakker OM, Tran HV, Robson AG, Lai-Cheong JE, Groves R, Holder GE, Moore AT. Angioid streaks with severe macular dysfunction and generalised retinal involvement due to a homozygous duplication in the ABCC6 gene. Eye. 2012;26:753-5.
$\boldsymbol{F}$

hyperautofluorescent ring and some discrete hyperautofluorescent lesions in close proximity to the ring). e, f Ultra-widefield autofluorescence $(532 \mathrm{~nm}$ ) imaging (Optos plc, Dunfermline, UK) acquired 9 years later

2. Cella W, Greenstein VC, Zernant-Rajang J, Smith TR, Barile G, Allikmets R, Tsang SH. G1961E mutant allele in the Stargardt disease gene ABCA4 causes bull's eye maculopathy. Exp Eye Res. 2009;89:16-24.

3. Burke TR, Fishman GA, Zernant J, Schubert C, Tsang SH, Smith RT, Ayyagari R, Koenekoop RK, Umfress A, Ciccarelli ML, Baldi A, Iannaccone A, Cremers FP, Klaver CC, Allikmets R. Retinal phenotypes in patients homozygous for the G1961E mutation in the ABCA4 gene. Invest Ophthalmol Vis Sci. 2012;53:4458-67.

4. Fujinami K, Sergouniotis PI, Davidson AE, Mackay DS, Tsunoda K, Tsubota K, Robson AG, Holder GE, Moore AT, Michaelides M, Webster AR. The clinical effect of homozygous ABCA4 alleles in 18 patients. Ophthalmology. 2013;120:2324-31.

5. Guymer RH, Héon E, Lotery AJ, Munier FL, Schorderet DF, Baird PN, McNeil RJ, Haines H, Sheffield VC, Stone EM. Variation of codons 1961 and 2177 of the Stargardt disease gene is not associated with age-related macular degeneration. Arch Ophthalmol. 2001;119:745-51.

6. Audo I, Vanakker OM, Smith A, Leroy BP, Robson AG, Jenkins SA, Coucke PJ, Bird AC, De Paepe A, Holder GE, Webster AR. Pseudoxanthoma elasticum with generalized retinal dysfunction, a common finding? Invest Ophthalmol Vis Sci. 2007;48:4250-6. 


\title{
Factors associated with pain during intravitreal injection of anti- vascular endothelial growth factor
}

\author{
Ngoc Chau Isabelle Nguyen ${ }^{1}$ Filippo Fabro ${ }^{1} \cdot$ Aude Ambresin $^{1} \cdot$ Sakina Ezziat $^{1} \cdot$ Ciara Bergin $^{1}$.

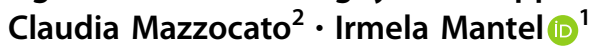

Received: 25 November 2017 / Revised: 19 March 2018 / Accepted: 22 March 2018 / Published online: 17 May 2018

(c) The Royal College of Ophthalmologists 2018

Intravitreal injection of anti-vascular endothelial growth factor (VEGF) is currently the standard-of-care treatment for various high-prevalence retinal disorders, including agerelated macular degeneration (AMD), diabetic macular oedema (DMO) and retinal vein occlusion (RVO). The injection is performed under topical anaesthesia, with some patients reporting discomfort during the injection, which could place a significant psychological burden in the long term.

According to the few reports that have investigated pain related to intraocular injections, pain scores are generally low $[1,2]$. However, the results relating to potentially associated factors, including age, gender [1], number of injections [1] and patient anxiety [2], are variable. In our study, we quantified the incidence and degree of injection-related pain, and investigated the associated risk factors.

We included 229 patients with painless retinal disease (AMD, DMO or RVO) who received intravitreal antiVEGF (ranibizumab, aflibercept or bevacizumab) injection with a $30 \mathrm{G}$ needle under topical anaesthesia (tetracaine $1 \%$ ). Before the injection, participants responded to a questionnaire about psychological pain-related elements [3]: general wellbeing, current level of anxiety, perceived quality of the information received and understanding of the treatment indication. The patients were Mean, pain during prior intravitreal injections was also asked. Each of these items was rated on a scale from 0 to 10 following the example of the Edmonton Symptom Assessment System [4]. Immediately after the procedure, participants

Irmela Mantel

irmela.mantel@fa2.ch

1 Department of Ophthalmology, University of Lausanne, Jules Gonin Eye Hospital, Fondation Asile des Aveugles,

Lausanne, Switzerland

2 Palliative and Supportive Care Service, Department of Medicine, University of Lausanne, Lausanne, Switzerland rated the degree of pain felt during the injection (scale 0 $10)$.

The results revealed pain in 132 participants (58\%), which was generally mild (mean pain score $1.7 \pm 2.0$, median 1.0), but severe (pain score 6-10) in 11 patients (5\%) (Fig. 1). Univariate analysis identified the following risk factors: preoperative anxiety $(p<0.01)$, pain during prior intravitreal injections $(p<0.01)$, chronic use of eye drops $(p=0.01)$ and quality of preoperative information provided ( $p=0.01$ ) (Table 1$)$. In multivariate analysis, the degree of anxiety $(p<0.01)$ and pain during prior injection $(p<0.01)$ were identified as independent factors (Table 1).

The role of anxiety has been reported [2], which might be related to the quality of preoperative information provided (a significant factor in univariate analysis). Measures that reduce perioperative stress (e.g. calming music) might be helpful [5]. However, we found that the surgeon's estimation of the patient's anxiety was a poor indicator of the actual anxiety level $\left(R^{2}=0.2\right)$. Thus, asking the patients about their anxiety would be more informative than relying on the surgeon's impressions.

Previous experience of painful intravitreal injections was an independent pain factor. It might be related to chronic irritation or mild subclinical inflammation of the eye. Thus, the patient's experience needs to be taken into account.

In conclusion, topical anaesthesia for intravitreal injections does not completely eliminate pain. The main independent factors are patient's anxiety and previous painful experiences. High-quality preoperative information, a reassuring surrounding and the use of relaxation technics [5] might be helpful, but this needs to be confirmed in further studies. 
Table 1 Results of the univariate and multivariate analyses of all included factors potentially associated with the degree of pain felt during the intravitreal injection procedure

\begin{tabular}{llll}
\hline Univariate analysis & $\begin{array}{l}\text { Correlation } \\
\text { coefficient }\end{array}$ & Confidence interval & Multivariate analysis \\
\hline$p$ & $\begin{array}{l}\text { Risk factor associated with } \\
\text { elevated pain }\end{array}$ & $p$
\end{tabular}

\section{Patient characteristics}

$\begin{array}{llll}\text { Age } & 0.39 & -0.01 & -0.03 \text { to } 0.01 \\ \text { Gender } & 0.15 & -0.39 & -0.91 \text { to } 0.14 \\ \text { History of intraocular surgery } & 0.89 & 0.04 & -0.49 \text { to } 0.56 \\ \begin{array}{lll}\text { Allergies } \\ \text { Allergies against drugs }\end{array} & 0.37 & 0.28 & -0.34 \text { to } 0.91 \\ \text { Other allergies } & 0.46 & 0.33 & -0.54 \text { to } 1.20 \\ \text { Any allergies } & 0.76 & 0.09 & -0.50 \text { to } 0.68\end{array}$

Systemic medications taken before injection

$\begin{array}{llll}\text { Analgesics } & 0.67 & 0.16 & -0.58 \text { to } 0.91 \\ \text { Tranquilisers } & 0.26 & 0.64 & -0.49 \text { to } 1.77\end{array}$

\section{Regular use of ocular drops}

Any ocular drops (including lubricants)

Prostaglandins

0.01 Regular use of ocular drops $\quad 0.78$

0.88
$-0.10$

0.22 to 1.34

-1.44 to 1.24

Self-reported psychological elements ranking outside the operating room (10-point scale)

$\begin{array}{lll}\text { General wellbeing } & 0.10 & 0.1 \\ \text { Level of anxiety } & 0.00 \text { Higher anxiety } & 0.1 \\ \begin{array}{l}\text { Quality of information about the } \\ \text { injection }\end{array} & 0.01 \text { Poorer information } & 0.1 \\ \begin{array}{l}\text { Appreciation and understanding } \\ \text { of treatment indication }\end{array} & 0.98 & 0.00\end{array}$

0.10

0.18

0.15

0.00

Surgeon's estimation of patient anxiety in the operating room (10-point scale)
0.74

Mean pain experienced during previous injections

\section{Redness of the eye}

Diffuse conjunctival hyperaemia

Focal episcleral hyperaemia

Ciliary hyperaemia
0.72

0.91

0.12

0.23

0.54

0.00

$-0.10$

0.07

0.02

0.27

0.00

0.23 injections

0.08 Presence of diffuse conjunctival hyperaemia

0.27

0.58
0.52

0.47

0.56
-0.02 to 0.23

0.09 to 0.28

0.00

0.03 to 0.27

-0.14 to 0.14

-0.10 to 0.14

-0.62 to 0.43

-1.15 to 1.29

-0.01 to 1.05

-1.09 to 1.62

-0.01 to 0.01

0.13 to 0.33

0.00

-0.05 to 1.09

0.41

-1.44 to 2.55

$V E G F$ vascular endothelial growth factor

\section{Compliance with ethical standards}

Conflict of interest The authors declare that they have no conflict of interest.

Informed consent Informed consent was obtained from all individual participants included in the study.
Research involving human participants All procedures performed in studies involving human participants were in accordance with the ethical standards of the institutional and/or national research committee and with the 1964 Helsinki declaration and its later amendments or comparable ethical standards. 
Fig. 1 Distribution of pain scores during the intravitreal injection procedure under topical anaesthesia, according to the patient's appreciation
Number of patients vs Score of pain

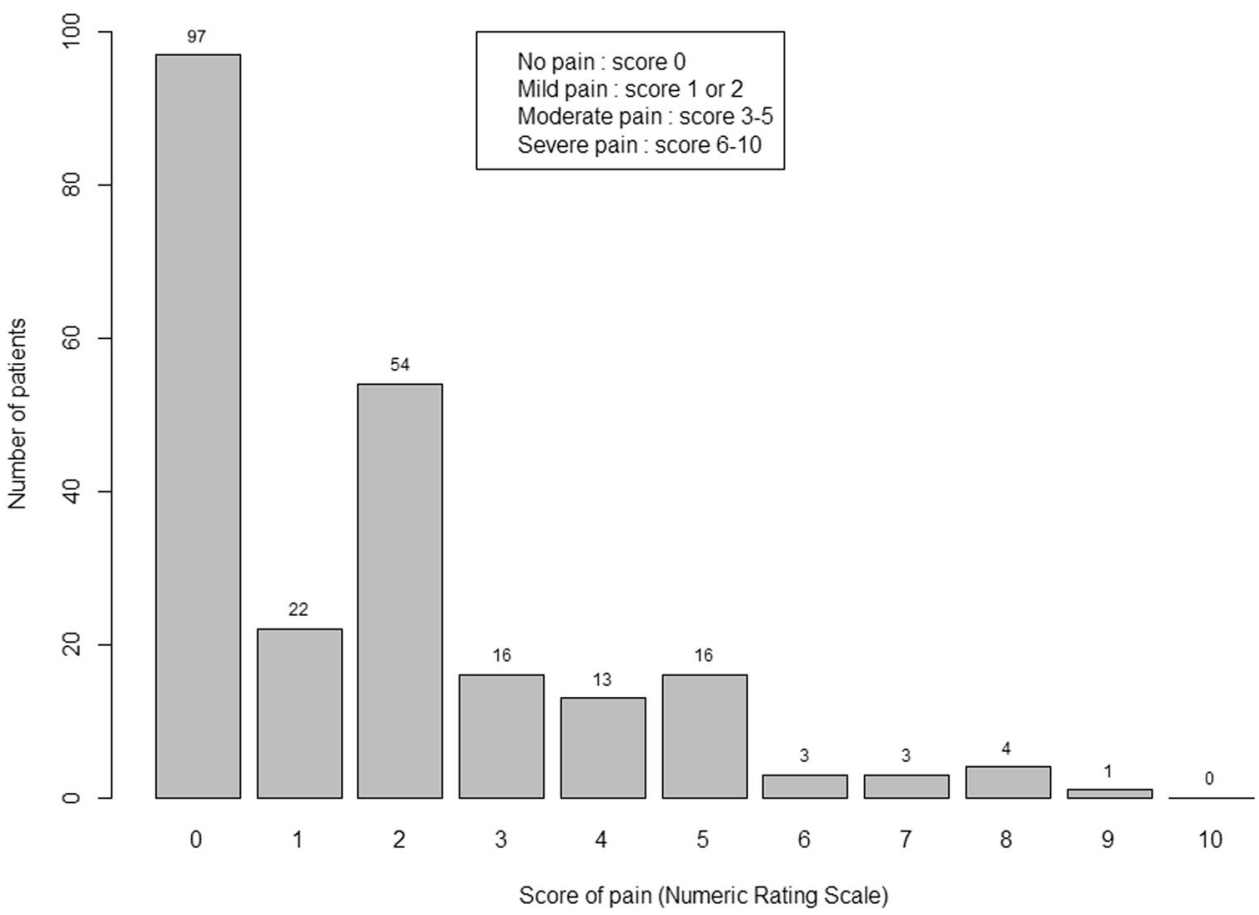

\section{References}

1. Rifkin L, Schaal S. Factors affecting patients' pain intensity during in office intravitreal injection procedure. Retina. 2012;32:696-700.

2. Segal O, Segal-Trivitz Y, Nemet AY, Cohen P, Geffen N, Mimouni M. Anxiety levels and perceived pain intensity during intravitreal injections. Acta Ophthalmol. 2016;94:203-4.

3. Okamura H, Fukui S, Nagasaka Y, Koike M, Uchitomi Y. Psychoeducational intervention for patients with primary breast cancer and patient satisfaction with information: an exploratory analysis. Breast Cancer Res Treat. 2003;80:331-8.

4. Nekolaichuk C, Watanabe S, Beaumont C. The Edmonton Symptom Assessment System: a 15-year retrospective review of validation studies (1991--2006). Palliat Med. 2008;22:111-22.

5. van der Heijden MJ, Oliai Araghi S, van Dijk M, Jeekel J, Hunink MG. The effects of perioperative music interventions in pediatric surgery: a systematic review and meta-analysis of randomized controlled trials. PLoS ONE. 2015;10:e133608.

\title{
Management of anterior capsular contraction syndrome: pitfall of circular capsulotomy technique with the neodymium YAG laser
}

\author{
Craig Wilde $^{1} \cdot$ Andrew Ross $^{1} \cdot$ Mary Awad ${ }^{1} \cdot$ Hean-Choon Chen ${ }^{2} \cdot$ Harminder S Dua $\mathbb{D}^{1}$
}

Received: 7 March 2018 / Accepted: 15 March 2018 / Published online: 18 May 2018

(c) The Royal College of Ophthalmologists 2018

\section{Harminder S Dua}

Harminder.Dua@nottingham.ac.uk

1 Ophthalmology and Vision Sciences, Division of Clinical Neurosciences, B Floor, EENT Centre, Queen's Medical Centre, University of Nottingham, Nottingham, UK

2 Royal Derby Hospital, Uttoxeter Road, Derby DE22 3NE, UK
Dear Editor,

Capsular contraction syndrome (CCS) is a distinct clinical entity associated with continuous curvilinear lcapsulorhexis (CCC) performed during phacoemulsificaion [1]. Although some degree of anterior capsular whitening and 
Fig. 1 a Mobile capsular fragment in the inferior $\mathrm{AC}$ of case 2 . The cornea was clear and there was no anterior chamber activity. b Anterior segment optical coherence tomogram of case 1 showing adhesion of the complete doughnut shaped capsular remnant to the corneal endothelium. c Diffuse slit lamp view of capsular fragment (arrows). d Post-YAG laser anterior capsulotomy showing the cut edge of the anterior capsule in a complete circular pattern, highlighted with a dashed line. The radial white lines illustrate the direction of the recommended radial cuts, perpendicular to the edge of the capsule margin
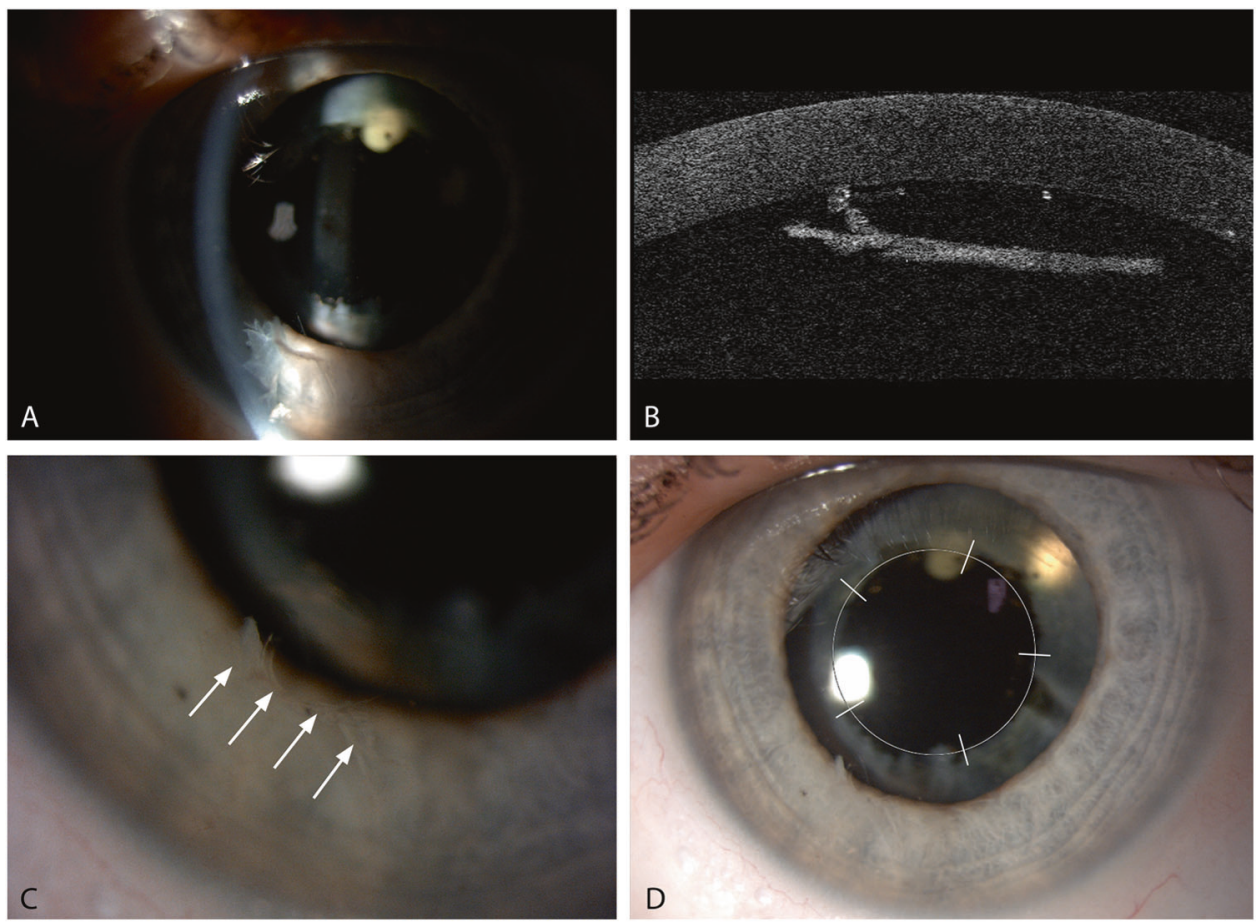

fibrosis is normal, extreme phimosis can become significant when it leads to visual axis opacification, secondary tilting, decentration, buckling, or posterior displacement of the intraocular lens (IOL) with hypermetropic shift [1]. The clinical impact is more with toric and multifocal lens implants. Extreme complications, such as lens dislocation, retinal, and ciliary body detachments can also occur [1].

The primary procedure employed to manage CCS is the use of Nd: YAG laser to create several radial incisions around the capsulorhexis margin [2], either in a linear or in a parabolic fashion. This releases the tension created by the phimosis, allowing the optic to return to its original position. Surgical approaches are numerous and include the use of a vitrector to create a circular capsular opening and removal of capsular and fibrotic remnants [3], along with intraocular diathermy [3] and incisions with microscissors [4]. A technique using straight scissors to cut the anterior capsule and subsequent completion of a CCC at the edge of the optic with forceps, using the force of adhesion between the anterior and posterior capsule has also been described [4].

Here we report two cases where anterior YAG laser capsulotomy was performed in a continuous circular fashion. The incised doughnut shaped fragment dropped into the anterior chamber (AC) (Fig. 1a). The first case was a woman aged 81 with posterior capsular opacification (PCO) and opacification of the anterior capsule with phimosis several years post phacoemulsification. She also had an idiopathic macular hole for which the capsulorhexis was enlarged to enhance the view during vitrectomy. The peripheral margin of the displaced remnant attached to the inferior corneal endothelium (Fig. 1b) and was fixed.

The second case was a 26-year-old female with a previous history of proliferative diabetic retinopathy treated with vitrectomy, endolaser and silicone oil fill. Subsequent cataract surgery combined with silicone oil removal resulted in CCS. YAG laser capsulotomy was performed in a circular fashion, creating a free-floating fragment (Fig. 1c). This settled in the inferior AC but changed position with head posture, obscuring vision during reading.

Both cases highlight problems of performing circular anterior capsulotomies. Free-floating remnants can be large and mobile, directly interfering with vision and pose risk of endothelial cell loss with associated corneal edema [5]. Their presence may necessitate surgical removal. Although there are no case series addressing risk of retained capsular remnants within the $\mathrm{AC}$, retained nuclear fragments pose substantial risk of corneal edema making their removal essential. The authors recommend YAG anterior capsulotomy in a spoke like pattern, radiating perpendicularly from the edge of the capsule margin, as illustrated (Fig. 1d). We recommend at least 4 initial cuts of $1 \mathrm{~mm}$, which can be extended further as required depending on the severity of the CCS and eccentricity of the capsule/intraocular lens complex. The cuts should generally not extend beyond the edge of the optic.

This method is safe and effective [2]. The effect is sometimes not instantaneous, but if this technique fails, further radial YAG laser capsulotomy or surgical approaches can be considered. Given the low incidence of CCS, 
training opportunities in its management are limited. Trainees should be aware of the different methods and their limitations.

\section{Compliance with ethical standards}

Conflict of interest The authors declare that they have no conflict of interest.

\section{References}

1. Davison JA. Capsule contraction syndrome. J Cataract Refract Surg. 1993;19:582-9.
2. Tsinopoulos I, Symeonidis C, Frangou E, Dimitrakos SA. Capsule contraction syndrome in eight cases of hydrophobic one-piece intraocular lens implantation. Clin Exp Optom. 2008;91:469-72.

3. Yeh PC, Goins KM, Lai WW. Managing anterior capsule contraction by mechanical widening with vitrector-cut capsulotomy. J Cataract Refract Surg. 2002;28:217-20.

4. Panagopoulos A, Chalioulias K, Kirkby GR. A new approach in the surgical management of anterior capsular phimosis syndrome. Ophthalmic Res. 2009;42:221-3.

5. Wilson RP, Gupta R. Focal corneal decompensation caused by an anterior capsulotomy remnant. J Cataract Refract Surg. 1997;23: $1273-4$.

\title{
Comment on: Assessment of patient-reported outcome and quality of life improvement following surgery for epiphora
}

\author{
Valerie Juniat $^{1} \cdot$ Sarju Athwal ${ }^{1} \cdot$ Adeela Malik $^{2} \cdot$ Mona Khandwala $^{1}$ \\ Received: 11 January 2018 / Accepted: 12 February 2018 / Published online: 18 May 2018 \\ (c) The Royal College of Ophthalmologists 2018
}

\section{Dear Editor,}

We read with great interest the article by Sipkova et al. [1] entitled "Assessment of patient-reported outcome and quality of life improvement following surgery for epiphora" in the December 2017 issue of Eye. We have reviewed the patient-reported outcomes of our own patients who have undergone surgery for epiphora. We would like to share our experience with readers.

Sipkova et al. [1] discussed the relevant limitations of the Glasgow Benefit Inventory (GBI), Munk symptoms score [2], and Lac-Q questionnaire. In our study, we prospectively enrolled 17 patients (20 eyes) who underwent lateral tarsal strip procedures for epiphora and lower lid laxity between June 2014 and September 2015. All 17 patients (20 eyes) completed the Munk score preoperatively and at 3 months

Valerie Juniat

vjuniat@doctors.org.uk

1 Maidstone and Tunbridge Wells NHS Trust, Hermitage Lane, Maidstone, Kent ME16 9QQ, UK

2 Moorfields Eye Hospital NHS Trust, 162 City Road, London EC1V 2PD, UK postoperatively. Patients subjectively scored their epiphora severity based on the $0-4$ scale, where. is no watering and. is constant watering. Statistical analysis was carried out using paired $T$-test. Average Munk score prior to lid surgery was $4.65 \pm 0.49 \mathrm{SD} \quad($ median $=5)$ and $2.05 \pm 1.05 \mathrm{SD}$ (median $=2$ ) postoperatively. The mean difference in preoperative and postoperative Munk score was $2.6 \pm 1.05 \mathrm{SD}$ (median $=3, p<0.001,95 \%$ CI 2.11-3.09). Only one patient had no change in their Munk score following lateral tarsal strip surgery, scoring. both pre and postoperatively.

Our study findings compare with those from Sipkova et al. [1], where their patients also showed significant improvement in Munk score following lid-tightening surgery $(p<0.001)$. In our experience, the Munk score can be applied realistically during busy outpatient clinical settings in view of its ease of completion. However, the Munk score has its limitations in assessing the impact of epiphora on specific daily tasks. For example, our single patient who did not show improvement in Munk score may have had very severe frequency of wiping from 50 times. day preoperatively (Munk score of 4) to 20 times. day postoperatively (Munk score of 4), an improvement for the patient. We therefore support Sipkova et al. [1] in their 
notion for the development of. validated PROM questionnaire for use following non-DCR, surgical intervention for epiphora.

\section{Compliance with ethical standards}

Conflict of interest The authors declare that they have no conflict of interest.

\section{References}

1. Sipkova Z, Vonica O, Olurin O, Obi EE, Pearson AR. Assessment of patient-reported outcome and quality of life improvement following surgery for epiphora. Eye. 2017;31:1664-71.

2. Munk PL, Lin DT, Morris DC. Epiphora: treatment by means of dacryocystoplasty with balloon dilatation of the nasolacrimal drainage apparatus. Radiology. 1990;177:687-90.

\title{
Exploring correlations between change in visual acuity following routine cataract surgery and improvement in quality of life assessed with the Glasgow Benefit Inventory
}

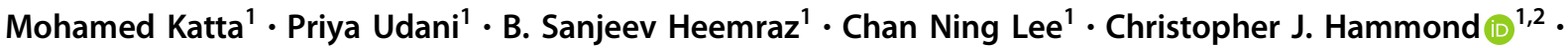 \\ Omar A. Mahroo $1,2,3,4$
}

Received: 15 January 2018 / Accepted: 25 April 2018 / Published online: 18 May 2018

(c) The Royal College of Ophthalmologists 2018

The Glasgow Benefit Inventory (GBI) [1] assesses patientperceived changes in quality of life following an intervention by means of 18 questions; the score ranges between a minimum of -100 (maximal detriment) to +100 (maximal benefit). As it is not procedure-specific, it can potentially be used to compare changes in quality of life following different interventions. Originally developed for use in otolaryngology, it has also been used in a range of oculoplastic procedures [2,3], and, more recently, we have deployed it after routine cataract surgery [4]. In the latter study, the questionnaire was administered to 109 patients, and significant improvement in quality of life was demonstrated with a positive GBI score.

Omar A. Mahroo

omar.mahroo@kcl.ac.uk

1 Department of Ophthalmology, St Thomas' Hospital, London SE1 7EH, UK

2 Department of Ophthalmology, King's College London, St Thomas' Hospital Campus, Westminster Bridge Rd, London SE1 7EH, UK

3 UCL Institute of Ophthalmology, University College London, 1143 Bath St, London EC1V 9EL, UK

4 Retinal Service, Moorfields Eye Hospital, 162 City Rd, London EC1V 2PD, UK
In the present study, we retrospectively retrieved visual acuity data for these patients (from the patient record) to explore correlation between change in visual acuity (decimalised) and GBI score. Patients for whom the preoperative or post-operative visual acuity was not available were excluded. 91 patients (83.5\%) were included: 48 were female; 53 were Caucasian, 30 Black, 3 Asian (5 had unrecorded ethnicity). The mean (SD) age was 71 (11) years.

Table 1 shows the main findings, and Fig. 1 plots GBI scores against change in visual acuity. For the whole cohort GBI score correlated positively with visual acuity with a Pearson correlation coefficient of $0.35\left(p=7 \times 10^{-4}\right)$. For first eyes, correlation was stronger than for second eyes. The visual acuity improvement for first eyes was greater than that for second eyes as might be expected (given the usual practice of operating on a worse seeing eye first). Importantly, an improvement in quality of life (positive GBI score) was seen after both first and second eye surgery, with no significant difference in scores, despite the difference in change in visual acuity. Mean pre and post-operative visual acuity did not differ significantly by ethnicity.

Our findings quantify the correlation between quality-of-life improvement, as measured by the GBI, and change in visual acuity. A significant correlation was seen. However, the strength of the correlation was 
Table 1 Change in visual acuity (VA) and Glasgow Benefit inventory (GBI) scores after first eye and second eye surgery

\begin{tabular}{|c|c|c|c|c|c|c|c|}
\hline & \multirow[t]{2}{*}{$n$} & \multicolumn{2}{|c|}{$\begin{array}{l}\text { Change in VA } \\
\text { (decimalised) }\end{array}$} & \multicolumn{2}{|l|}{ GBI score } & \multirow[t]{2}{*}{ Correlation coefficient } & \multirow[t]{2}{*}{ Significance $(p)$} \\
\hline & & Mean (SD) & $95 \% \mathrm{CI}$ & Mean (SD) & $95 \% \mathrm{CI}$ & & \\
\hline 1 st eye & 57 & $0.44(0.37)$ & $0.34-0.54$ & $+23.6(18.2)$ & $18.8-28.5$ & 0.53 & $2 \times 10^{-5}$ \\
\hline 2nd eye & 34 & $0.22(0.32)$ & $0.11-0.33$ & $+22.4(23.4)$ & $14.2-30.6$ & 0.10 & 0.57 \\
\hline All patients & 91 & $0.36(0.37)$ & $0.28-0.44$ & $+23.2(20.2)$ & $19.0-27.4$ & 0.35 & $7 \times 10^{-4}$ \\
\hline
\end{tabular}

Pearson coefficients are given for correlation between change in VA and GBI score. 95\% CI, 95\% confidence interval for mean. The change in visual acuity was greater for first eyes compared with second eyes ( $p=0.005$, unpaired $t$ test), but GBI scores for first eyes and second eyes were similar $(p=0.68)$, indicating similar benefits in quality of life for first eyes and second eyes

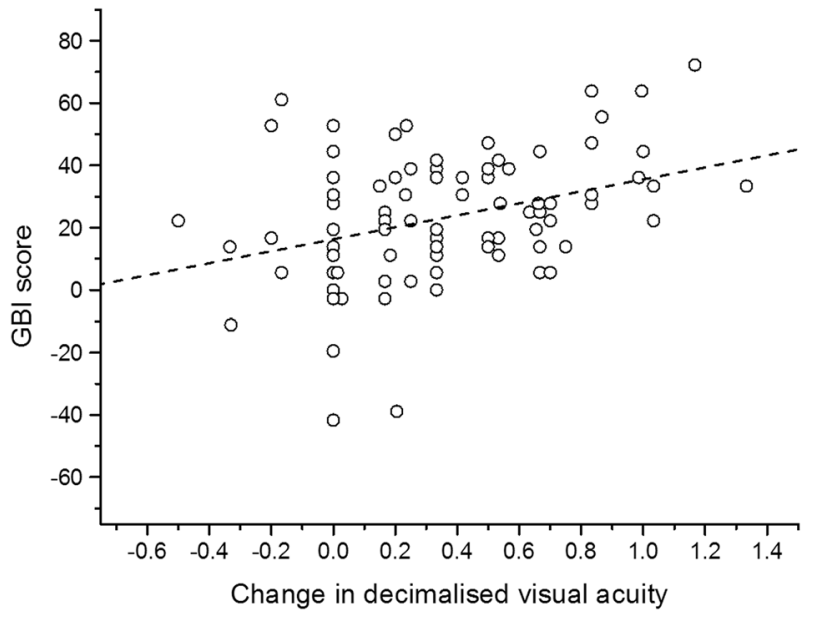

Fig. 1 GBI score plotted against change in decimalised visual acuity. The dashed line provides a simple linear fit. Correlation was found to be significant $\left(p=7 \times 10^{-4}\right)$

moderate to low overall, which supports the notion that change in visual acuity alone does not capture the full improvement in quality of life conferred by cataract surgery. This is especially true for second eyes. The improvement in visual acuity was less than that for first eyes, but the perceived improvement in quality of life appeared to be just as strong, suggesting that this is driven by factors other than visual acuity (possibly improved stereopsis or reduced anisometropia as well as other less quantifiable factors). Thus our findings confirm that the benefit of cataract surgery is not assessable by visual acuity alone, particularly in the case of second eyes.

Funding OAM receives support from the Wellcome Trust (Grant 206619_Z_17_Z), Fight for Sight, and the NIHR Biomedical Research Centre at Moorfields Eye Hospital and the UCL Institute of Ophthalmology.

\section{Compliance with ethical standards}

Conflict of interest The authors declare that they have no conflict of interest.

\section{References}

1. Robinson K, Gatehouse S, Browning GG. Measuring patient benefit from otorhinolaryngological surgery and therapy. Ann Otol Rhinol Laryngol. 1996;105:415-22.

2. Smith HB, Jyothi SB, Mahroo OA, Shams PN, Sira M, Dey S, et al. Patient-reported benefit from oculoplastic surgery. Eye. 2012;26: 1418-23.

3. Mahroo OA, Hysi PG, Dey S, Gavin EA, Hammond CJ, Jones CA. Outcomes of ptosis surgery assessed using a patient-reported outcome measure: an exploration of time effects. Br J Ophthalmol. 2013;98:387-90.

4. Heemraz BS, Lee CN, Hysi PG, Jones CA, Hammond CJ, Mahroo OA. Changes in quality of life shortly after routine cataract surgery. Can J Ophthalmol J Can d'ophtalmologie. 2016;51:282-7. 